\title{
Efeito da equoterapia no desempenho funcional de crianças e adolescentes com autismo
}

\section{Effect of hippotherapy on functional performance of children and teens with autism}

\author{
Daniele Dornelles Bender ${ }^{1}$, Nicole Ruas Guarany ${ }^{2}$ \\ http:/dx.doi.org/10.11606/issn.2238-6149.v27i3p271-277
}

Bender DD, Guarany NR. Efeito da equoterapia no desempenho funcional de crianças e adolescentes com autismo. Rev Ter Ocup Univ São Paulo. 2016 set.-dez.;27(3):271-7.

RESUMO: A equoterapia vem sendo utilizada como tratamento reabilitador em diferentes patologias, no entanto, sua eficácia ainda não é comprovada no que tange ao desempenho funcional de indivíduos com autismo. O objetivo deste estudo é identificar o efeito da equoterapia no desempenho funcional de crianças e adolescentes com autismo comparando praticantes e não praticantes. Participaram do estudo indivíduos com autismo com idades entre 3 e 15 anos, ambos os sexos divididos entre Praticantes e Não Praticantes, emparelhados por gênero e idade. Utilizou-se um questionário para avaliar variáveis socioeconômicas e para avaliação do desempenho funcional o Inventário de Avaliação Pediátrica de Incapacidade (PEDI) e a Medida de Independência Funcional (MIF), aplicados aos cuidadores, para $<8$ anos e $>8$ anos, respectivamente. Foram entrevistados 28 cuidadores (14 em cada grupo). Houve diferença para o desempenho funcional das crianças que praticavam equoterapia avaliados pelo PEDI na área de Autocuidado ( $p=0,041)$ e Mobilidade $(p=0,001)$. Não houve diferença para o desempenho funcional daqueles avaliados pela MIF ( $p=0,384)$. Este estudo sugere que a equoterapia é eficaz para crianças com autismo, nas tarefas das áreas de mobilidade e autocuidado, sendo uma área inovadora para terapia ocupacional.

DESCRITORES: Autismo, Terapia Assistida por Cavalos; Terapia Ocupacional.
Bender DD, Guarany NR. Effect of hippotherapy on functional performance of children and teens with autism. Rev Ter Ocup Univ São Paulo. 2016 Sept.-Dec.;27(3):271-7.

ABSTRACT: Hippotherapy has been used as rehabilitative treatment, however, its effectiveness has not yet proven in regard to functional performanceof individuals with autism. The aim of this study is to identify the effect of hippotherapy on functional performance in children and adolescents with autism compared practitioners and non-practitioners. Study participants were individuals with autism aged 3 to 15 years, both genders divided between Practitioners and Non-Practitioners, matched by gender and age. It was used a questionnaire to assess socioeconomic variables and to make the assessment of the functional performance Pediatric Evaluation of Disability Inventory (PEDI) and the Functional Independence Measure (FIM), applied to caregivers, to $<8$ years and $>8$ years, respectively. There were interviewed 28 caregivers (14 in each group), there was difference in functional performance of children practicing hippotherapy reviews by area PEDI Self-care $(p=0.041)$ and mobility $(p=0.001)$. There was no difference in the functional performance of those assessed by the FIM $(p=0.384)$. This study suggests that hippotherapy is effective for children with autism, the tasks in the areas of mobility and self-care, being an innovative area for occupational therapy.

KEYWORDS: Autism, Equine-Assisted Therapy, Occupational Therapy.

Este estudo é parte integrante de Trabalho de Conclusão de Curso apresentado ao Curso de Terapia Ocupacional da Universidade Federal de Pelotas, como requisito parcial para a obtenção do título de Terapeuta Ocupacional. O estudo não está sendo avaliado para publicação em outra revista e não foi apresentado em eventos. Este estudo não obteve financiamento de agência de fomento.

1. Graduada em Terapia Ocupacional pela Universidade Federal de Pelotas. Terapeuta Ocupacional do Hospital Escola da Universidade Federal de Pelotas

2. Doutora em Ciências Médicas pela Universidade Federal do Rio Grande do Sul. Docente do curso de Terapia Ocupacional da Universidade Federal de Pelotas.

Endereço para correspondência: Avenida Princesa do Sul, 2948, Três Vendas, Pelotas-RS.: nicolerg.ufpel@yahoo.com.br 


\section{INTRODUÇÃO}

Os transtornos do espectro do autismo (TEA) são um grupo de desordens complexas ${ }^{1}$, o diagnóstico é feito a partir de prejuízos em dois principais eixos, os déficits sociais e de comunicação e os comportamentos repetitivos e restritivos ${ }^{2}$. Esses sinais e sintomas devem levar a dificuldades adaptativas importantes dos sujeitos estando presentes, geralmente, antes dos três anos de idade ${ }^{3}$.

Diversos estudos tentam elucidar a etiologia do autismo, embora ainda não exista um consenso entre os autores ${ }^{4}$, estudos atuais têm demonstrado que o autismo é multifatorial e depende de componentes genéticos ${ }^{5}$ e ambientais $^{6}$. Ocorre predominantemente em meninos na proporção de 4 meninos para uma menina,é frequente a ocorrência do autismo entre irmãos ${ }^{7}$.

As características clínicas do autismo podem interferir em diferentes áreas de ocupação, tais como as atividades de vida diária (AVD) e instrumentais de vida diária, lazer, sono e descanso, no brincar, na escola, na participação social e no trabalho ${ }^{1}$. A Terapia Ocupacional considera os diferentes tipos de ocupação nas quais os clientes podem se envolver, a fim de possibilitar maior participação e desempenho nessas atividades ${ }^{8}$.

O questionamento a respeito da etiologia do autismo e da eficácia das várias opções de tratamento aplicáveis à clínica, incluindo aqueles que ainda têm pouca evidência científica, leva as famílias a uma busca incansável por intervenções que, se não comprovadas cientificamente, podem ser dispendiosas e nem sempre bem sucedidas ${ }^{9}$.

Definida pela Associação Nacional de Equoterapia (ANDE) como um método terapêutico e educacional, a equoterapia utiliza-se do cavalo como recurso terapêutico e tem como objetivo o desenvolvimento biopsicossocial dos sujeitos ${ }^{10}$. A prática há muito é utilizada como recurso em diferentes condições clínicas, incluindo-se indivíduos com autismo, deficiência visual, Síndrome de Down e paralisia cerebral ${ }^{11}$.

No entanto, é consenso entre os pesquisadores da temática que a eficácia da Equoterapia como recurso terapêutico para o tratamento desses indivíduos e os seus resultados no desempenho funcional sejam evidenciados e comprovados a fim de contribuir para o embasamento científico dos profissionais ${ }^{12,13}$, uma vez que são escassos estudos científicos relacionados ao assunto, principalmente no Brasil, dificultando a realização de intervenções apropriadas que apresentem resultados significativos e que se tornem uma opção viável para os cuidadores e também para os serviços de saúde ${ }^{11}$.
Nesta perspectiva, este estudo teve como objetivo identificar o efeito da equoterapia no desempenho funcional de crianças e adolescentes com autismo, através da comparação entre praticantes e não praticantes.

\section{PROCEDIMENTOS METODOLÓGICOS}

Trata-se de um estudo transversal comparativo de caráter quantitativo. A amostra foi composta por indivíduos com autismo, sendo o diagnóstico clinico dos participantes estabelecidos previamente por médicos especialistas (Neuropediatras, Pediatras e Geneticistas), de acordo com prontuários disponibilizados nos locais onde o estudo foi realizado. Os participantes possuíam idades entre 3 e 15 anos, ambos os sexos, sendo metade da amostra de indivíduos praticantes de equoterapia provenientes do Centro de Equitação e Equoterapia Zeca Macedo na cidade de Rio Grande-RS e da Associação de Pais e Amigos dos Excepcionais-APAE na cidade de PelotasRS. Os não praticantes, eram provenientes do Núcleo de Neurodesenvolvimento Professor Mário Coutinho da Universidade Federal de Pelotas (UFPel) e do Centro de Atendimento ao Autista, ambos na cidade de Pelotas. O emparelhamento da amostra nos dois grupos deu-se através do gênero e idade, podendo o não praticante ter um ano a mais ou a menos que o praticante. Somaram-se a estes critérios de inclusão estar realizando ou não atendimentos com outros profissionais (Fonoaudiologia, Terapia Ocupacional, Psicopedagogia, entre outros) e o aceite em participar do estudo através da assinatura do Termo de Consentimento Livre e Esclarecido. Foram excluídos indivíduos que apresentavam algum outro diagnóstico clínico associado (Síndrome de Down, Paralisia Cerebral, Epilepsia, etc.). Optou-se pela aplicação dos instrumentos aos cuidadores pois as características clínicas do autismo poderia dificultar a compreensão e fornecimento de respostas adequadas.

Foi utilizado um questionário fechado elaborado pelas autoras para a coleta das seguintes variáveis: Gênero, Renda Familiar, Escolaridade do Cuidador, Tempo de Prática Equoterápica (para os praticantes) e outros atendimentos realizados. Para a avaliação do desempenho funcional foram utilizados o Inventário de Avaliação Pediátrica de Incapacidade (PEDI) ${ }^{14}$ para crianças até 7 anos e 6 meses e a Medida de Independência Funcional $(\mathrm{MIF})^{15}$ para pacientes com idade superior.

$\mathrm{O}$ instrumento PEDI fornece informações quantitativas sobre o desenvolvimento infantil e o processo de aquisição das habilidades funcionais e independência necessária para o desempenho de atividades e tarefas da 
rotina diária das crianças com idades entre 6 meses e 7 anos e 6 meses, é dividido em três partes distintas, sendo que cada parte inclui três áreas de desempenho: autocuidado, mobilidade e função social ${ }^{14}$. O instrumento MIF avalia de forma quantitativa os cuidados demandados por uma pessoa para a realização de tarefas motoras e cognitivas de vida diária. São avaliadas atividade de autocuidado, transferências, locomoção, controle esfincteriano, comunicação e cognição social ${ }^{15}$.

Os escores padrão dos instrumentos foram utilizados conforme manual de aplicação, sendo que para o PEDI utilizou-se para comparação entre praticantes e não praticantes apenas o escore padronizado normativo que fornece informações sobre o desempenho esperado de crianças brasileiras com a mesma faixa etária com o desenvolvimento normal. Para MIF foi utilizada a pontuação total sem subdividir entre área de mobilidade e área cognitiva.

A análise estatística foi realizada através do programa Statistical Package for Social Sciences Release (SPSS 16.0) for Windows (SPSS, 2008). As variáveis foram descritas na forma de frequência simples, medianas e intervalos interquartis. O teste de Mann-Whitney foi utilizado para comparar o desempenho funcional dos indivíduos com autismo que praticam e não praticam equoterapia. $\mathrm{O}$ valor se significância para este estudo foi de $\mathrm{p}<0,05$.

Esta pesquisa foi submetida à Plataforma Brasil e autorizada pelo Comitê de Ética e Pesquisa da Universidade Federal de Pelotas por meio do CAAE: 26641714.8.0000.5317.

\section{RESULTADOS E DISCUSSÃO}

As entrevistas ocorreram entre os meses de março a maio de 2014, durante esse período foram entrevistados um total de 28 cuidadores de crianças e adolescentes com autismo. Primeiramente foram entrevistados os cuidadores dos indivíduos que realizavam a prática de equoterapia. Neste grupo, não houve recusa e foram excluídos dois indivíduos que não preencheram os critérios de inclusão, sendo entrevistados um total de 14 cuidadores. Concluída esta etapa, iniciaram-se as entrevistas com os cuidadores dos indivíduos que não realizavam a prática, estas foram realizadas na sala de espera dos locais quando da possibilidade de emparelhamento dos sujeitos e do preenchimentos dos critérios de inclusão.

Em ambos os grupos o sexo masculino foi o mais frequente $(92,9 \%)$ (Tabela 1), o que demonstra que a amostra está de acordo com a literatura atual, que evidencia que o autismo é mais comum em indivíduos do sexo masculino em uma proporção de $4: 1^{1,4}$.

Tabela 1 - Caracterização da Amostra

\begin{tabular}{l|c|c}
\hline Variáveis & $\begin{array}{c}\text { Praticantes } \\
(\mathbf{n}=\mathbf{1 4})\end{array}$ & $\begin{array}{c}\text { Não Praticantes } \\
(\mathbf{n}=\mathbf{1 4})\end{array}$ \\
\hline Gênero & $13 / 14$ & $13 / 14$ \\
Masculino & $1 / 14$ & $1 / 14$ \\
Feminino & 7,14 & 6,93 \\
\hline Idade (md) & $(3-15$ anos $)$ & $(3-15$ anos $)$ \\
Mín/max & & \\
\hline Renda & $1 / 14$ & $1 / 14$ \\
$<1$ SMN & $4 / 14$ & $12 / 14$ \\
De 1 a 3 SMN & $9 / 14$ & $1 / 14$ \\
$>$ 3 SMN & & \\
\hline Escolaridade & - & $2 / 14$ \\
Não alfabetizado & $1 / 14$ & $7 / 14$ \\
Até 4 anos & $4 / 14$ & $3 / 14$ \\
Até 8 anos & $3 / 14$ & $2 / 14$ \\
Até 11 anos & $6 / 14$ & - \\
$>11$ anos & & \\
\hline Tempo de Prática & $2 / 14$ & - \\
$<6$ meses & $4 / 14$ & - \\
De 6 meses a 1 ano & $8 / 14$ & - \\
$>1$ ano &
\end{tabular}

*SMN: Salário Mínimo Nacional vigente neste período, equivalente a $\mathrm{R} \$ 724,00$.

No que tange a idade dos participantes do estudo, o GP apresentou mediana de idade de 7,14 anos, o GNP apresentou mediana de 6,93 anos de idade (Tabela 1).

Em relação à renda e escolaridade dos cuidadores no GP a maior parte da amostra $(n=9)$ possuía renda superior a três Salários Mínimos Nacionais (SMN), e mais de 11 anos de estudo $(n=6)$. Para o GNP a renda da maior parte da amostra $(n=12)$ é de um a três SMN e até 4 anos de estudo $(n=7)$. O teste de Mann-Whitney identificou diferença significativa entre os grupos GP e GNP para Renda Familiar ( $\mathrm{p}=0,006)$ e Escolaridade dos Pais $(\mathrm{p}=0,001)$ (Tabela 1$)$.

$\mathrm{Na}$ literatura atual é possível identificar estudos que relatam que o aumento da escolaridade acarreta em maior concentração de renda entre a população $0^{16}$, fator que reflete tambémno acesso aos serviços de saúde, a maior concentração de renda permite facilitação no acesso aos serviços de saúde, enquanto a baixa concentração dificulta o acesso aos serviços. Incluso nesses serviços, a Equoterapia, que, embora tenha se consolidado nos últimos anos, ainda não é acessível à população como um todo, devido ao custo elevado da prática e a escassez do serviço em alguns locais/regiões do país ${ }^{17}$. 
No GP, os cuidadores relataram que os indivíduos realizavam atendimentos de Fonoaudiologia $(\mathrm{n}=7)$, Psicopedagogia $(\mathrm{n}=9)$, Musicoterapia $(\mathrm{n}=4)$, Pedagogia $(n=3)$ e Psicologia $(n=1)$, Escola Especial $(n=3)$ e Escola Regular $(n=4)$. No GNP os indivíduos realizavam atendimentos de Fonoaudiologia $(n=3)$, Terapia Ocupacional $(\mathrm{n}=10)$, Psicopedagogia $(\mathrm{n}=3)$, Musicoterapia $(n=1)$, Pedagogia $(n=5)$ e Educação Física $(n=1)$, Escola Especial $(n=3)$ e Escola Regular $(n=9)$

Com relação ao grupo que realiza a prática equoterápica, a maior parte $(n=8)$ realiza a prática há mais de 1 ano (Tabela 1).

O teste de Mann-Whitney identificou diferença estatisticamente significativa para o desempenho funcional das crianças até 7 anos e 6 meses que praticavam equoterapia avaliados pelo PEDI para a área de Autocuidado $(\mathrm{p}=0,041)$ e Mobilidade $(\mathrm{p}=0,001)$, a área de função social $(p=0,103)$ não apresentou significância estatística.

Com relação ao desempenho funcional das crianças avaliadas pelo instrumento PEDI, foi possível observar que o GP apresentou escores mais elevados em duas áreas: Autocuidado $(18,0)$ e Mobilidade $(34,0)$ (Figura 1).

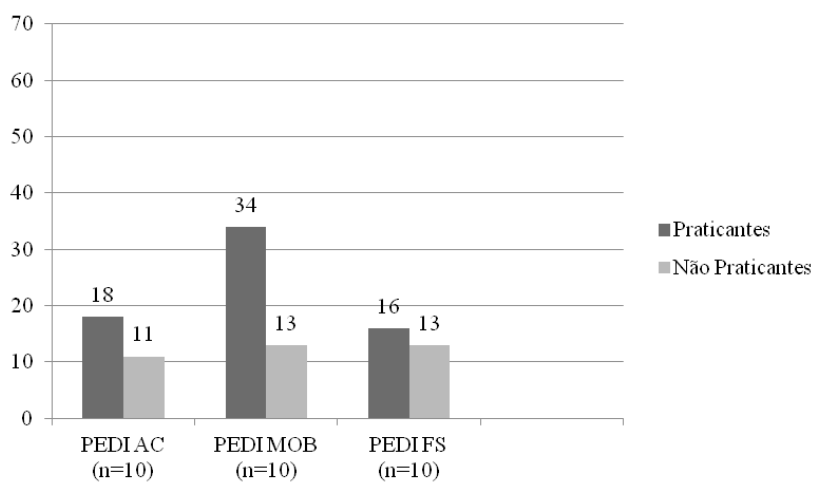

Figura 1 - Comparativo entre GP e GNP através do Inventário de Avaliação Pediátrica de Incapacidade (PEDI)

Pontuação do PEDI para as áreas de Autocuidado (AC), Moblidade (MOB), Função Social (FS):

$<30$ pontos: Atraso de desenvolvimento ou desempenho significativamente inferior ao normal.

30 à 70 pontos: Atraso de desenvolvimento considerado dentro do padrão de normalidade

50 pontos: Escore médio esperado para faixa etária

$>70$ pontos: Desenvolvimento ou desempenho significativamente superior ao normal
Esta análise descritiva indica que tanto o GP quanto o GNP, embora tenham apresentado escores diferentes em algumas áreas, apresentam um atraso no desenvolvimento bastante significativo nas três áreas avaliadas. Fato este que se deve, provavelmente, às próprias características clínicas do autismo relacionadas às áreas do desempenho funcional avaliadas neste estudo.

A área de autocuidado do PEDI, onde são mensuradas habilidades de alimentação, cuidado pessoal, vestir, banho e uso de toalete, com maiores pontuações para o GP, sugere que a Equoterapia apresenta resultados positivos para os indivíduos com autismo na realização das AVD. Deve-se também levar em consideração o maior grau de escolaridade dos cuidadores do GP, podendo este ser um fator que influencia no melhor desempenho nessas atividades. A escassez de bibliografia é um fator limitante para confirmar esses dados, os poucos estudos já publicados evidenciam melhora nas habilidades de autocuidado como resultados secundários das intervenções em equoterapia que tem como foco melhora nas habilidades motoras e/ ou sociais ${ }^{18,19}$.

O Terapeuta Ocupacional é o profissional reconhecido legalmente para avaliar e intervir nas tarefas de autocuidado, na equoterapia o cavalo atua como um motivador $^{20}$, facilitando a participação do indivíduo nos cuidados com o animal durante as sessões, tal como a alimentação (como por exemplo, fornecendo cenouras), participar da escovação da crina do animal, utilizando pente/escova, cuidados com a aparência (embelezamento do cavalo), participar da atividade de banho do animal, entre outras, estimulando dessa forma a aquisição de conceitos e habilidades necessárias para as atividades na área de autocuidado.

A área de mobilidade do teste PEDI que avalia transferências e mobilidade em ambientes internos e externos também apresentou maiores pontuações para o GP. Atualmente, embora ainda não inclusos nos Manuais de Diagnóstico, muitos estudos têm evidenciado que déficits motores estão presentes em indivíduos com autismo, cerca de $80 \%$ destes sujeitos apresentam algum déficit motor tais como, na coordenação motora ampla e fina, equilíbrio, força, agilidade, alterações nos padrões de marcha e tônus muscular ${ }^{1,21,22}$.

A estimulação das habilidades motoras é fundamental e proporciona além da melhora dos aspectos motores, possibilidade de maior participação em outras atividades, assim como as atividades de vida diária, de lazer e na interação social dos indivíduos com autismo ${ }^{22}$. Deste modo torna-se fundamental que a estimulação motora 
seja iniciada precocemente, e a equoterapia, de acordo com a literatura, consolida-se como um bom recurso a ser utilizado com essa clientela no que tange a aquisição de habilidades motoras, funcionando não só como um agente facilitador durante as atividades, mas utilizandose das potencialidades do cavalo como estimulador dos componentes motores ${ }^{23,24,11,18,19}$.

$\mathrm{Na}$ área de Função Social, porém, os resultados encontrados não apresentaram diferenças estatisticamente significativas entre o GP e GNP, nessa área são avaliadas capacidades de comunicação, interação social, tarefas domésticas e da comunidade. No autismo há um grande comprometimento com relação às habilidades sociais, sendo esta uma das áreas problema para o estabelecimento do diagnóstico ${ }^{1,2}$. Os prejuízos nessa área podem ser identificados através da dificuldade na interação social, comportamento social inadequado, dificuldade em manter contato visual, dificuldade em compreender a comunicação social do outro e no estabelecimento de atividades em grupo ${ }^{1}$. Os estudos atuais demonstram que a equoterapia apresenta resultados positivos com relação à Função Social tais como adequação no humor, melhora do contato visual, linguagem expressiva/conversa, cuidados com os animais, melhora nos comportamentos sociais, aumento da volição e interesse por novas tarefas ${ }^{25,26,18-20}$, porém informações sobre as sessões e quais as atividades realizadas para alcançar esses resultados são insuficientes e vagas, dificultando a compreensão deste processo. $\mathrm{O}$ autismo é caracterizado por uma falta de compreensão social humana, enquanto cavalos são animais altamente sociáveis que irão responder a estímulos humanos muito sutis, essa capacidade de resposta é importante para a aquisição do conceito de causa e efeito possibilitando a aprendizagem concreta dos indivíduos com autismo, aumentando a compreensão do impacto do seu comportamento na comunicação social ${ }^{20}$.

Acredita-se que os atendimentos grupais apresentam-se como uma alternativa para alcançar resultados significativos com essa clientela, sugerindo avanços com relação à ansiedade, hiperatividade, problemas de comportamento, resolução de problemas e melhora nas habilidades sociais ${ }^{27}$, pois é no contexto das relações sociais que emergem a linguagem, o desenvolvimento cognitivo, o autoconhecimento e o conhecimento do outro ${ }^{28}$.

$\mathrm{O}$ teste de Mann-Whitney não identificou diferença estatisticamente significativa para o desempenho funcional das crianças e adolescentes com idades acima de 7 anos e 6 meses entre os grupos GP e GNP avaliados pela MIF ( $p=0,384)$ (Figura 2). Ambos os grupos apresentaram necessidade de $25 \%$ de assistência para a realização das atividades avaliadas. A MIF avalia os cuidados demandados por uma pessoa para a realização de tarefas motoras e cognitivas de vida diária. São avaliadas atividade de autocuidado, transferências, locomoção, controle esfincteriano, comunicação e cognição social.

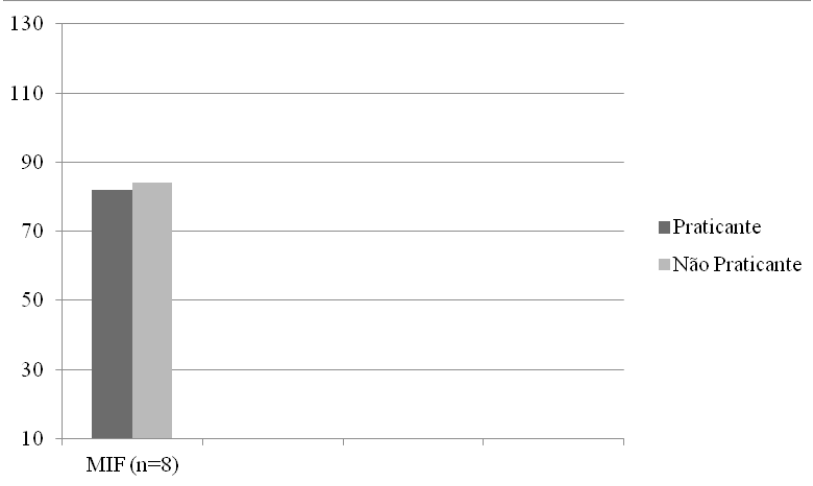

Figura 2 - Comparativo entre GP e GNP através da Medida de Independência Funcional (MIF)

18 pontos: Dependência Completa (assistência total)

19-60 pontos: Dependência Modificada (assistência em até 50\% das tarefas)

61-103 pontos: Dependência Modificada (assistência em até 25\% das tarefas)

104-126 pontos: Independência Completa ou Modificada

As hipóteses levantadas de acordo com esses resultados relacionam-se a alguns pontos específicos como um possível diagnóstico tardio desses sujeitos, conforme a literatura, a agilidade no diagnóstico tem aumentado a cada ano, porém ainda é possível identificar dificuldades para o diagnóstico precoce ${ }^{29,30}$. Esse fato acarreta em demora para acessar serviços que possam promover melhora nos comportamentos/dificuldades característicos do autismo antes de se tornarem estruturados. Associando este dado aos nossos resultados, sugere-se que a prática equoterápica pode ter iniciado tardiamente neste grupo e que a mudança nas áreas avaliadas não tenha sido significativa após a prática. Outro fator que também pode estar relacionado, diz respeito a idade dos participantes, sugerindo que quanto maior a idade mais homogênea a população nos dois grupos. Uma outra hipótese é que talvez o instrumento MIF não seja adequado para avaliar a funcionalidade de crianças e adolescentes com autismo por não conseguir captar especificidades da clínica desses sujeitos. 


\section{CONSIDERAÇÕES FINAIS}

Este estudo verificou que a equoterapia apresentase como um método terapêutico eficaz para os indivíduos com autismo para o ganho na área de autocuidado e mobilidade, uma vez que apresentou resultados estatisticamente significativos para as crianças menores de 8 anos. Acredita-se que na área de função social resultados positivos possam ser obtidos com a implementação de abordagens grupais nos programas de intervenção na Equoterapia, assim como sugere a literatura atual.

Sugere-se que novos estudos sejam realizados com crianças maiores de 8 anos com a aplicação de outro instrumento mais específico e que seja sensível as alterações no desenvolvimento para comparar com os dados deste estudo.

O presente estudo apresenta algumas limitações a serem corrigidas em estudos posteriores, tais como

\section{REFERÊNCIAS}

1. Tuchman R, Rapin I, org. Autismo: abordagem neurobiológica. Porto Alegre: Artmed, 2009.

2. American Psychiatric Association. Diagnostic and statistical manual of mental disorders. 5th ed. Arlington, VA: American Psychiatric Publishing; 2013

3. Klin A. Autismo e síndrome de Asperger: uma visão geral. Rev Bras Psiquiatr. 2006;28(Sup1):S3-S11. DOI: http://dx.doi.org/10.1590/S1516-44462006000500002

4. Fombonne E. The epidemiology of autism: a review. Psychological Med. 1999;29(4):769-86. DOI: http://dx.doi. org/10.1017/S0033291799008508

5. 'Gupta AR, State MW. Autismo: genética. Rev Bras Psiquiatr. [Internet]. 2006;28(Supl 1):S29-S38. DOI: http://dx.doi.org/10.1590/S1516-44462006000500005

6. 'Alison MC, Jo W, Reiko G. Factors that influence autism knowledge in hispanic cultures: a pilot study. J Racial Ethn Health Disparities. 2016:1-9, DOI: http://dx.doi.org/10.1007/ s40615-016-0213-4

7. Mecca TP, Bravo RB, Velloso RL, Schwartzman JS, Brunoni D, Teixeira MCTV. Rastreamento de sinais e sintomas de transtornos do espectro do autismo em irmãos. Rev Psiquiatr. Rio Gd. Sul, 2011;33(2):116-20. DOI: http://dx.doi. org/10.1590/S0101-81082011000200009

8. Cavalcanti A, Dutra FCM, Elui VMC. Estrutura da prática da terapia ocupacional: domínio e processo - 3a ed. Rev Ter a utilização de um número amostral maior e aplicação de instrumentos eficazes na distinção de gravidade do autismo a fim de possibilitar um emparelhamento mais equânime entre os grupos de praticantes e não praticantes. Outro fator que consideramos como limitação, diz respeito às diferenças culturais entre todos os participantes, principalmente aqueles de cidades distintas. Acredita-se na importância de que novos estudos com esta mesma temática sejam realizados a fim de evidenciar os benefícios da equoterapia para os indivíduos com autismo.

Os resultados deste estudo foram apresentados em todos os locais onde realizou-se a coleta dos dados, afim de efetivar o início da prática de Equoterapia o mais precoce possível para a obtenção de melhores resultados. Também foram apresentados os resultados dos instrumentos de avaliação PEDI e MIF de cada participante para uma possível intervenção multiprofissional nas áreas que apresentaram déficits importantes.

Ocup Univ São Paulo. 2015;26(Esp):1-49.DOI: http://dx.doi. org/10.11606/issn.2238-6149.v26iespp1-49

9 Lanning BA, Baier ME, Ivey-Hatz J, Krenek N, Tubbs JD.Effects of Equine Assisted Activities on Autism Spectrum Disorder. J Autism Dev Disord. 2014; 44(8):1897-907. DOI: http://dx.doi.org/10.1007/s10803-014-2062-5.

10. ANDE/BRASIL, Fundamentos básicos sobre equoterapia. In: $1^{\circ}$ Congresso Brasileiro de Equoterapia, 1999. Anais. Brasília, DF; 1999. p.13-16.

11. Severo JT. Org. Equoterapia: equitação, saúde e educação. $1^{\text {a }}$ Ed. São Paulo: Senac; 2010.

12. Bass MM, Duchowny CA, Llabre MM. The effect of therapeutic horseback riding on social functioning in children with autism. J Autism Develop Disorders. 2009;39(9):1261-7. DOI: http://dx.doi.org/10.1007/s10803-009-0734-3

13. Kern JK, Fletcher CL, Garver CR, Mehta JA, Grannemann BD, Knox KR, Richardson TA, Trivedi $\mathrm{MH}$. Prospective trial of equine-assisted activities in autism spectrumdisorder. Alternative Ther Health Med. 2011;17(3):14-20. Disponível em: https://www.researchgate. net/publication/51874213_Prospective_Trial_of_Equineassisted_Activities_in_Autism_Spectrum_Disorder

14. Mancini MC, Haley, SM. Inventário de avaliação pediátrica de incapacidade (PEDI): manual da versão brasileira adaptada. Ed: UFMG, 2005. 
15. Riberto M, Miyazaki MH, Jucá SSH, Sakamoto H, Pinto PPN, Battistella LR. Validação da versão brasileira da Medida de Independência Funcional. Rev Acta Fisiatr. 2004;11(2):72-6. Disponível em: http:/www.unifra.br/professores/anabonini/ Valida\%C3\%A7\%C3\%A3o\%20Brasileira\%20MIF.pdf.

16. Salvato MA, Ferreira PCG, Duarte, AJM. O impacto da escolaridade sobre a distribuição de renda. Est Econ. (São Paulo), 2010;40(4):753-91. DOI: https://dx.doi.org/10.1590/ S0101-41612010000400001

17. Travassos C, Castro MSM. Determinantes e desigualdades sociais no acesso e na utilização de serviços de saúde. In: Políticas e sistema de saúde no Brasil. Fiocruz, p. 183-206, 2012.

18. Ajzenman HF, Standeven JW, Shurtleff TL. Effect of hippotherapy on motor control, adaptive behaviors, and participation in children with autism spectrum disorder: a pilot study. Am J Occup Ther. 2013;67(6):653-63. DOI: http://dx.doi.org/10.5014/ajot.2013.008383

19. O'haire ME. Animal-assisted intervention for autism spectrum disorder: A systematic literature review. J Autism Develop Disorders. 2013,43(7):1606-22. DOI: http://dx.doi. org/10.1007/s10803-012-1707-5

20. Gabriels RL, Agnew JA, Holt KD, Shoffner A, Zhaoxing P, Ruzzano S, Clayton GH, Mesibov G. Pilot study measuring the effects of therapeutic horseback riding on school-age children and adolescents with autism spectrum disorders. Autism Spectrum Disorders. 2012,6(2):578-88. DOI: http://dx.doi.org/10.1016/j.rasd.2011.09.007

21. Anderson-Hanley C, Tureck K, Schneiderman RL. Autism and exergaming: effects on repetitive behaviors and cognition. Psychol Res Behavior Manag. 2011;4:129-37. DOI: http://dx.doi.org/10.2147/PRBM.S24016

22. Hilton CL, Cumpata K, Klohr C, Gaetke S, Artner A, Johnson H, Dobbs S. Effects of exergaming on executive function and motor skills in children with autism spectrum disorder: a pilot study. Am Jo Occup Ther. 2014,68(1):57-65. DOI: http://dx.doi.org/10.5014/ ajot.2014.008664

Recebido em: 25.04.16

Aceito em: 04.11.16
23. Lermontov T. A psicomotricidade na equoterapia. 1.ed. São Paulo: Ideias e Letras, 2004.

24. Bruzek, CE. Socioemotional Benefits of Animal-Assisted Occupational Therapy with Children: A Review of the Literature and Directions for Future Research.Tese (Honors Program University) University of South Florida St. Petersburg, 2014. Disponível em: http://dspace.nelson. usf.edu/xmlui/bitstream/handle/10806/10007/USFSP\%20 Honors \%20 Thesis\%20-\%20 Christine\%20 Bruzek. pdf? sequence $=1$

25. Taylor RR, Kielhofner G, Smith C, Butler S, Cahill SM, Ciukaj MD, Gehman M. Volitional change in children with autism: a single-case design study of the impact of hippotherapy on motivation. Occup Ther Mental Health. 2009;25(2):192-200. DOI: http://dx.doi. org/10.1080/01642120902859287

26. Ward SC, Whalon K, Rusnak K, Wendell K, Paschall N.The Association between therapeutic horseback riding and the social communication and sensory reactions of children with autism. J Autism Develop Disorders. 2013;43(9):2190-8. DOI: http://dx.doi.org/10.1007/s10803-013-1773-3

27. Epp KM. Outcome-based evaluation of a social skills program using art therapy and group therapy for children on the autism spectrum. Children Schools. 2008;30(1):27-36. DOI: http://dx.doi.org/10.1093/cs/30.1.27

28. Moura MLS. A interação social e solução de problemas por crianças: questões metodológicas, resultados empíricos e implicações educacionais. Temas Psicol. 1993;1(3):39-48. Disponível em: http://pepsic. bvsalud.org/scielo.php? script $=$ sci_arttext\&pid $=$ S1413389X1993000300006\&lng=pt.

29. Valicenti-McDermott M, Hottinger K, Seijo R, Shulman L. Age at diagnosis of autism spectrum disorders. J Pediatrics. 2012;161(3):554-6. DOI: http://dx.doi. org/10.1016/j.jpeds.2012.05.012

30. Daniels AM, Mandell DS. Explaining differences in age at autism spectrum disorder diagnosis: A critical review. Autism. 2014;18(5):583-97. DOI: $10.1177 / 1362361313480277$ 